\title{
A case of concurrent deep venous thrombosis, pseudoaneurysm, and extremity abscess in an intravenous methamphetamine abuser
}

BY MATTHEW L HARRISON

\begin{abstract}
Introduction. Intravenous drug abuse is a global concern with an estimated 16 million people engaging in this behavior worldwide. (1) In addition, Methamphetamine abuse is widely reported with 1.2 million Americans declaring its use in 2012. (2)

Case description. The patient presented to the Emergency Department for "right groin pain" at a site she had been injecting methamphetamine. She had a palpable, non-pulsatile mass of her right groin, diffuse erythema and tenderness of her right leg, and several ecchymotic areas on multiple toes. She was found to have an extensive deep vein thrombosis of the right lower extremity, right femoral pseudoaneurysm, septic emboli, and deep space infection of her right groin. The pseudoaneurysm was removed surgically, an extra-anatomical arterial bypass was placed, an inferior vena caval filter was inserted, anticoagulants were initiated, the groin abscess was debrided and washed out, and the patient was started on antibiotics.
\end{abstract}

Discussion. Emergency physicians practicing clinically should be aware of the 
potential adverse outcomes of intravenous drug abuse. This patient encounter details nearly all of the significant adverse outcomes patients could experience: cellulitis, deep space infection/abscess, deep venous thrombosis, arterial pseudoaneurysm, and septic emboli.

Key words: abscess, venous thrombosis, false aneurysm, pseudoaneurysm, methamphetamine, intravenous substance abuse, intravenous drug abuse

\section{Introduction}

Injecting drug use is a moderately prevalent practice that is recognized globally, with recent studies estimating approximately 16 million people engage in this behavior worldwide. (1) In addition, 1.2 million Americans reported using Methamphetamine in 2012, including 133,000 first time users. (2) This report details some of the negative effects that intravenous (IV) Methamphetamine abuse can cause.

\section{Case description}

The patient was a 45 year old white female who presented to the Emergency Department on July 30, 2012 with the chief complaint of "right groin pain." The pain had started nearly one month prior as a mild ache, but had substantially increased in severity over the last week. In addition, over the last week she noticed a developing mass in her right inguinal area that had increased in size since initial presentation. She now also complained of right lower extremity swelling, redness, and "blackened areas" on multiple toes of her right foot. She had presented to the Emergency Department six days earlier, but left without being seen because "the wait was too long." She had returned to the ED one day later, been evaluated, and prescribed Cephalexin for "inguinal lymphadenitis." She states her symptoms had not improved with treatment, but had worsened, necessitating this visit.

Her past medical, surgical, and family histories were negative for disease. She was an active smoker, used marijuana regularly, and admitted to a greater than 20 year history of IV Methamphetamine abuse. She also related that after several years of IV Methamphetamine injections, she had exhausted the veins of her 
bilateral upper extremities, and now was only able to inject the veins of her lower extremities, principally her femoral veins bilaterally. She lived alone. Upon review of systems, she complained of chills without objective fever, and right lower extremity pain. Otherwise her review of systems was negative.

With the exception of resting tachycardia at a rate of 116 beats per minute, her vital signs were within normal limits. Upon examination, she was a middle aged Caucasian female, who appeared older than her stated age. She was in moderate distress secondary to pain, and was moaning and writhing around on the hospital gurney. Examination of her right leg revealed an exquisitely tender, erythematous, non-pulsating mass in her inguinal crease measuring six centimeters in width and four centimeters in length. The remainder of her right leg was edematous, erythematous, and mildly tender to palpation. In addition, her right foot was cooler to touch than her left foot, and pulses could only faintly be palpated at the dorsalis pedis artery. Her right, second through fifth toes had dark discolorations and developing bullae, consistent with ischemia/emboli. In the affected toes, there was no sign of capillary refill.

Labs were ordered, including a complete blood count with differential, complete metabolic panel, blood cultures, lactic acid, serum pregnancy test, and coagulation studies. In addition, parenteral opioid analgesia and an antiemetic were administered, and a computed tomography scan (CT) of her pelvis with intravenous contrast material was ordered. On laboratory evaluation, her white blood cell count was found to be fifteen thousand, with a significant left shift. She was moderately anemic, and had a slight elevation of her liver function tests, but had otherwise unremarkable labs, as well as a negative pregnancy test. A hepatitis panel, obtained later in her hospital course, revealed anti-Hepatitis C antibodies. CT revealed an approximately 3.0 x 2.2 centimeter $(\mathrm{cm})$ pseudoaneurysm of the common femoral artery, probable deep vein thrombosis of the right femoral vein and right external iliac vein, as well as soft tissue edema and fat stranding of the distal pelvis and proximal right thigh with reactive groin lymph nodes, consistent with deep space infection and overlying cellulitis (figures 1, 2). Subsequent venous duplex examination confirmed the right common femoral deep venous thrombosis.

Broad spectrum antibiotics were administered, and the Hospitalist service was contacted for admission to the hospital. Vascular Surgery was consulted, and the patient was taken to the operating room (OR) for groin exploration, and irrigation. 
Upon operative exploration, a large inguinal abscess was discovered containing greater than 100 milliliters of purulent material. This was evacuated, and a sample was sent for culture. It and the blood cultures eventually grew Methicillin Resistant Staphylococcus Aureus. An intraoperative venogram revealed extensive right sided deep vein thrombosis, for which an inferior vena caval filter was placed, and anticoagulants were initiated post-surgery. In addition, a femoral artery pseudoaneurysm measuring $3 \mathrm{~cm}$ long by $5 \mathrm{~cm}$ wide was discovered, and excised. Due to the extensive infectious arterial damage sustained, an extraanatomical arterial bypass was created using an artificial graft coupled with the patient's superficial femoral artery. A wound vacuum was placed to allow continued drainage. She tolerated the surgery well, and was returned to the intensive care unit afterward.

The patient spent 16 days in the hospital receiving IV antibiotics and wound care. She was discharged home, to continue oral Clindamycin for four weeks, as well as oral anticoagulants. She was scheduled for follow up appointments with Vascular Surgery for discontinuation of her wound vacuum, as well as her primary care physician for anticoagulation monitoring. She was counseled for drug and smoking cessation, and was scheduled for follow up with Psychiatry as well.

\section{Discussion}

Most patients likely will not experience all of the negative sequela of IV drug use concurrently. However, equipped with the knowledge of the possible ramifications of such abuse, physicians will be more adept at examining their unique patient, and discerning which diagnostic tests to order. Emergency physicians practicing clinically should be aware of the potential negative outcomes of IV drug abuse. This patient encounter details nearly all of the significant adverse outcomes patients could experience: cellulitis, deep space infection/abscess, deep venous thrombosis, arterial pseudoaneurysm, and septic emboli.

Figure 1. Computed tomography scan (coronal view) revealing a right femoral artery pseudoaneurysm, with surrounding deep space infection/abscess. 


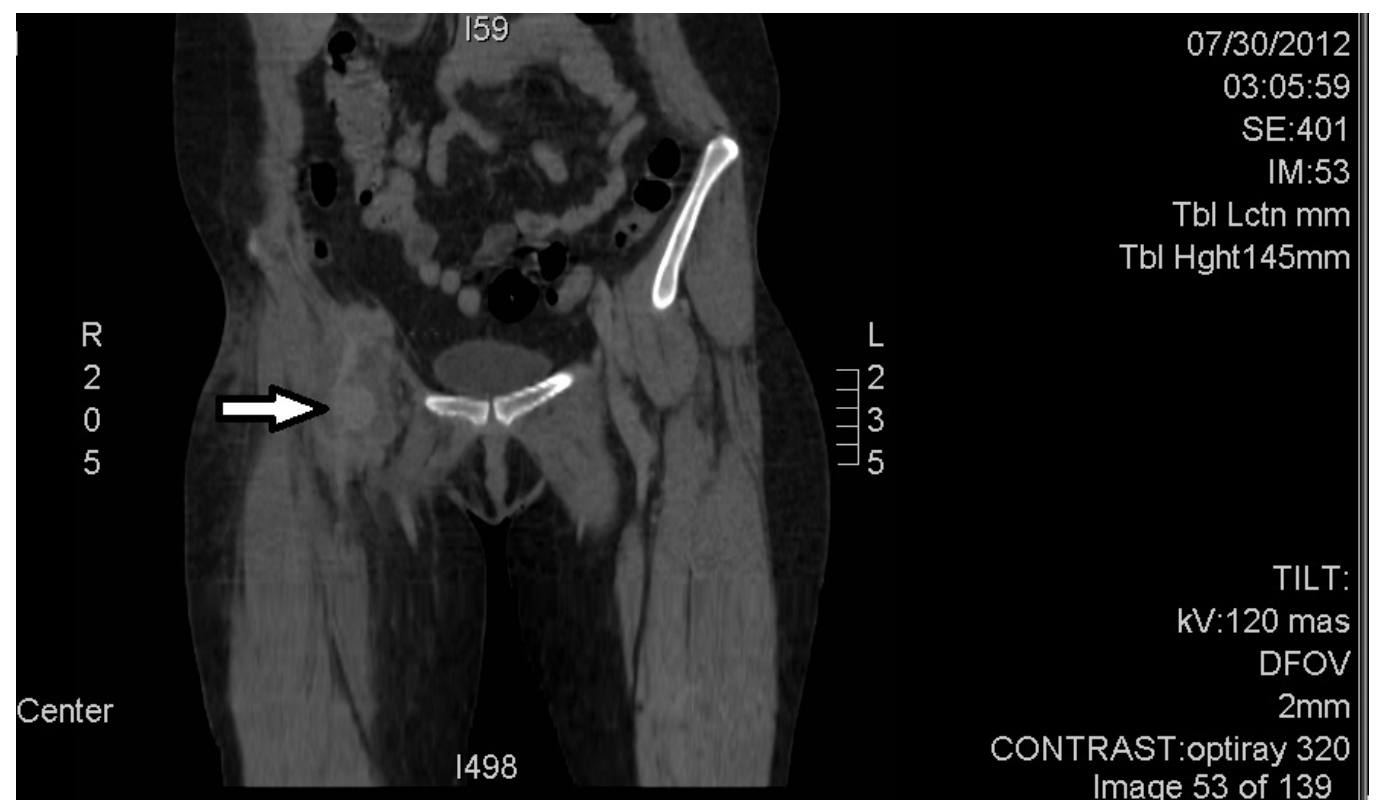

Figure 2. Computed tomography scan (transverse view) revealing right inguinal abscess.

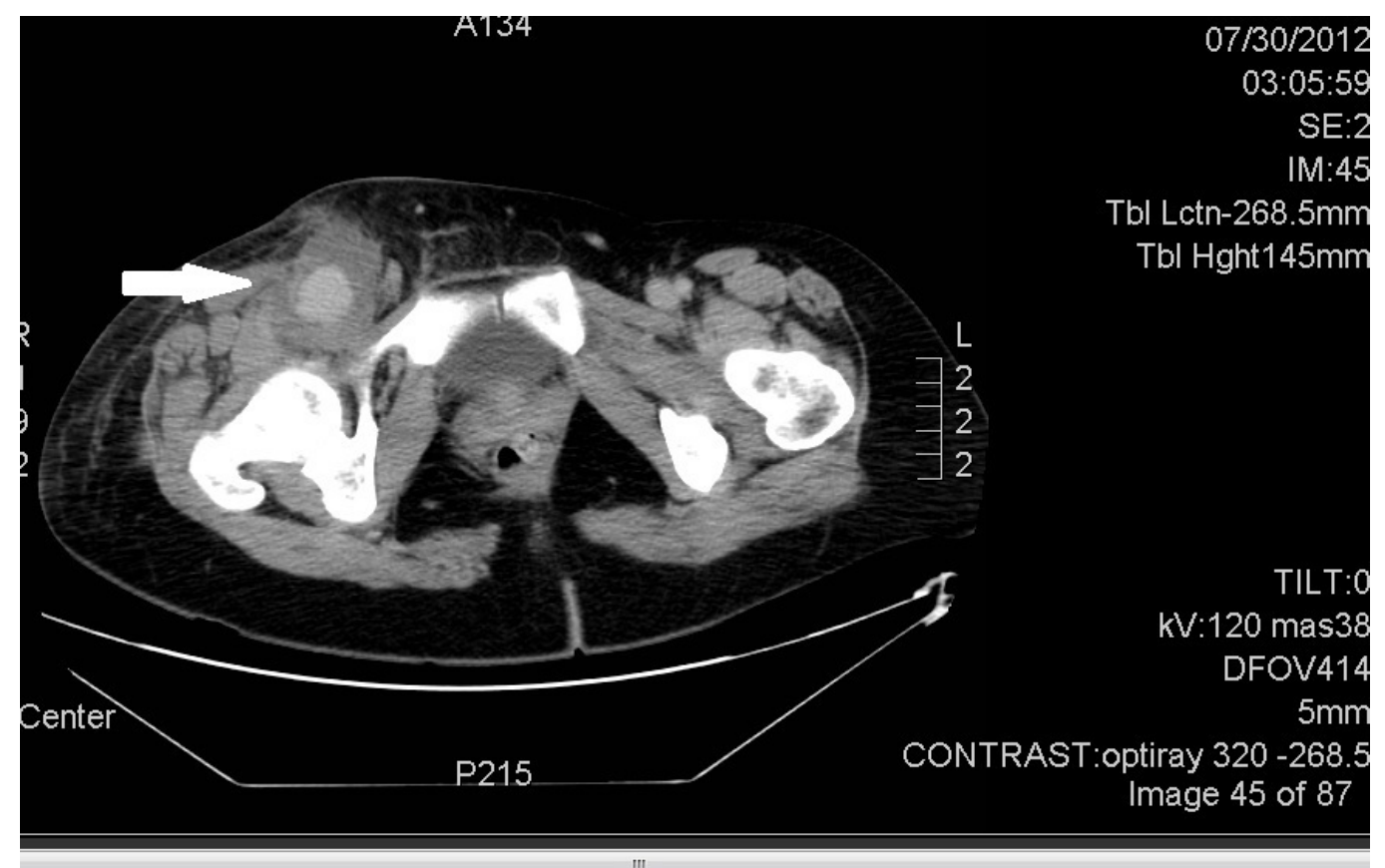

\section{References}

1. Mathers BM, Degenhardt L, Phillips B, Wiessing L, Hickman M, Strathdee SA. Global epidemiology of injecting drug use and HIV among people who inject 
drugs: a systematic review. Lancet 2008;372(9651):1733-45.

2. Substance Abuse and Mental Health Services Administration, Results from the 2011 National Survey on Drug Use and Health: Summary of National Findings, NSDUH Series H-44, HHS Publication No. (SMA) 12-4713. Rockville, MD: Substance Abuse and Mental Health Services Administration, 2012.

\author{
Matthew L Harrison \\ Texas Health Resources \\ Huguley Hospital Fort Worth South \\ 11801 South Freeway, Burleson, Texas 76028 , USA \\ Phone: $(817) 944-3627$ \\ Fax: (817) $568-3340$ \\ E-mail: matthewlharrison@gmail.com
}

